\title{
Stationary distribution of the surplus process in a risk model with a continuous type investment
}

\author{
Yang Hyeon $\mathrm{Cho}^{a}$, Seung Kyoung $\mathrm{Choi}^{a}$, Eui Yong Lee ${ }^{1, a}$ \\ ${ }^{a}$ Department of Statistics, Sookmyung Women's University, Korea
}

\begin{abstract}
In this paper, we stochastically analyze the continuous time surplus process in a risk model which involves a continuous type investment. It is assumed that the investment of the surplus to other business is continuously made at a constant rate, while the surplus process stays over a given sufficient level. We obtain the stationary distribution of the surplus level and/or its moment generating function by forming martingales from the surplus process and applying the optional sampling theorem to the martingales and/or by establishing and solving an integro-differential equation for the distribution function of the surplus level.
\end{abstract}

Keywords: risk model, surplus process, stationary distribution, integro-differential equation, martingale, optional sampling theorem

\section{Introduction}

We consider a continuous time surplus process in a risk model which involves a continuous type investment. The surplus, with initial level of $u_{0}>0$, linearly increases at a constant rate $c>0$ due to the incoming premium. Meanwhile, claims arrive according to a Poisson process of rate $\lambda>0$ and decrease the level of the surplus jump-wise by random amounts which are independent and identically distributed with distribution function $G$ of mean $\mu>0$. If the surplus process reaches a sufficient level $V>u_{0}$, the investment of the surplus to other business is continuously made at a constant rate $a(0<a<c)$ until the surplus process goes below $V$. The next investment starts if the surplus process goes over $V$ again.

It is usually assumed that $c$ is larger than $\lambda \mu$, the expected total amount of claims per unit time, however, $c-a$ is assumed to be less than $\lambda \mu$, in this paper, to keep the surplus process from being infinitely large. To analyze stochastically the level of the surplus in an infinite horizon, we also assume that the surplus process continues to move even though the level of the surplus becomes negative. In the classical risk model, we stop the surplus process and say that a ruin occurs if the level of the surplus goes below 0 .

In practice, an insurance company does not allow the surplus of a policy to get infinitely large, since the large stock of the surplus increases the opportunity cost. The insurance company hardly stops managing or operating the policy even though the surplus of the policy is exhausted. Hence, our assumptions are very practical. The concept of the investment of the surplus was introduced by Jeong et al. (2009) and Jeong and Lee (2010). They studied some optimal policies related to managing the surplus in the risk model.

\footnotetext{
${ }^{1}$ Corresponding author: Department of Statistics, Sookmyung Women's University, Cheongpa-ro 47-gil 100, Yongsan-gu, Seoul 04310, Korea. E-mail: eylee@ sookmyung.ac.kr

Published 30 September 2016 / journal homepage: http://csam.or.kr

(c) 2016 The Korean Statistical Society, and Korean International Statistical Society. All rights reserved.
} 
The classical risk model has been studied by many authors by assuming that a ruin occurs if the surplus becomes negative. They have studied the ruin probability of the surplus and some interesting characteristics, such as the time of ruin, the surplus before ruin and the deficit at ruin. The core result on the ruin probability is well summarized in Klugman et al. (2004). The first passage time of the surplus to a certain level was firstly introduced by Gerber (1990), thereafter, Gerber and Shiu (1997) obtained the joint distribution of the time of ruin, the surplus before ruin and the deficit at ruin. Dickson and Willmot (2005) calculated the density of the time of ruin by inverting its Laplace transform.

Dufresne and Gerber (1991) generalized the classical risk model by assuming that the surplus is perturbed by diffusion process between the time epochs of occurrence of claim and studied the ruin probability of the surplus. Won et al. (2013) generalized the risk model of Dufresne and Gerber (1991) by assuming that there are two types of claim, where one occurs more frequently but the size is smaller than the other, and obtained the ruin probability of the surplus. Song et al. (2012) generalized the classical risk model by assuming that the premium rate depends on the current level of the surplus and obtained the joint distribution of the time of ruin, the surplus before ruin and the deficit at ruin.

However, most works, in the above, have been concentrated on the ruin probability of the surplus and its related characteristics, until Cho et al. (2013) analyze some transient and stationary behaviors of the surplus process in the risk model with investments. Thereafter, Kim and Lee (2015) adopted a level crossing approach to obtain the stationary distribution of the surplus process in the risk model with dividends and reinvestments. The concepts of dividend and investment keep the surplus process from being infinitely large, and hence, enable us to find the stationary behavior of the surplus process. However, in the previous models including Jeong et al. (2009) and Jeong and Lee (2010), either the investment or the dividend was made immediately, jump-wise and by fixed amounts.

In this paper, by assuming that the investment of the surplus to other business is continuously made at a constant rate while the surplus process stays over a sufficient level $V$, we study the stationary distribution of the surplus process. In Section 2.1, we study the stationary distribution of the surplus level while the surplus process being over $V$, by forming martingales from the surplus process and applying the optional sampling theorem to the martingales. In Section 2.2, we obtain the moment generating function of the stationary distribution of the surplus level while the surplus process being under $V$, by establishing an integro-differential equation for the distribution function of the surplus level and solving the equation. In Section 2.3, we, finally, show how to combine the results in Sections 2.1 and 2.2 to obtain the stationary distribution of the whole surplus process.

The surplus process in our risk model with a continuous type investment is illustrated in Figure 1, where $U(t)$ is the level of the surplus at time $t \geq 0, \times$ denotes the time epoch where the claim occurs, and $Y$ is the random variable representing the amount of a claim.

\section{Stationary distribution of the surplus process}

Let $F(x, t)$ be the distribution function of $U(t)$, the surplus at time $t \geq 0$, that is,

$$
F(x, t)=P\{U(t) \leq x\}, \quad \text { for }-\infty<x<\infty .
$$

Let $F(x)$ be the stationary distribution function of $U(t)$, that is,

$$
F(x)=\lim _{t \rightarrow \infty} F(x, t), \text { for }-\infty<x<\infty .
$$

In this section, we obtain $F(x)$. To do that, we, first, decompose $\{U(t), t \geq 0\}$ into two processes $\left\{U_{1}(t), t \geq 0\right\}$ and $\left\{U_{2}(t), t \geq 0\right\}$. $U_{1}(t)$ is formed by separating the periods where $U(t) \geq V$ from 


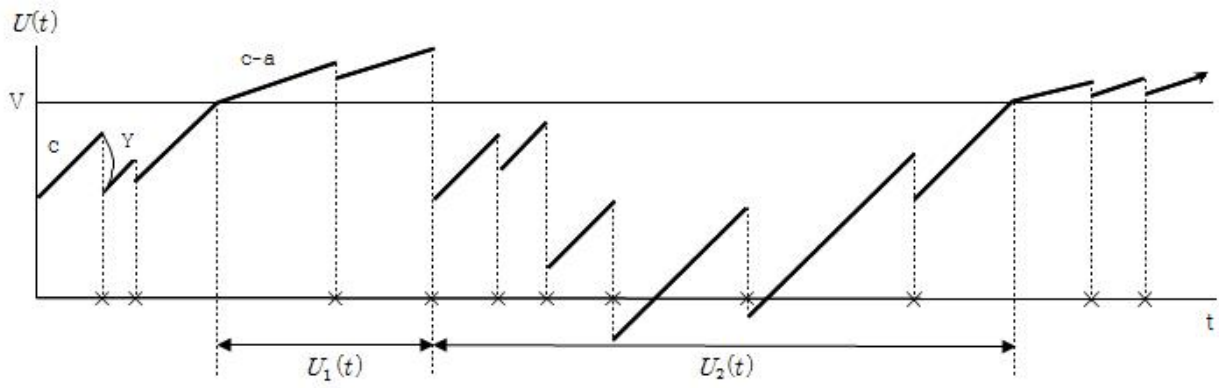

Figure 1: A sample path of $U(t)$.

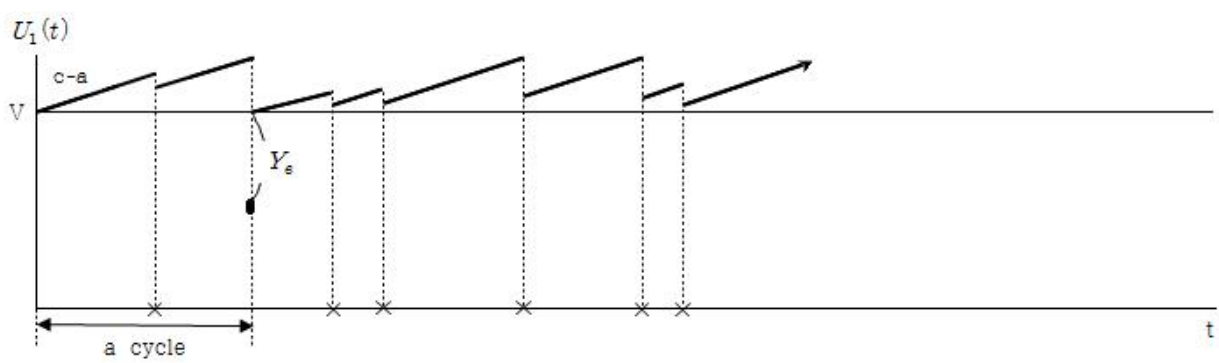

Figure 2: A sample path of $U_{1}(t)$.

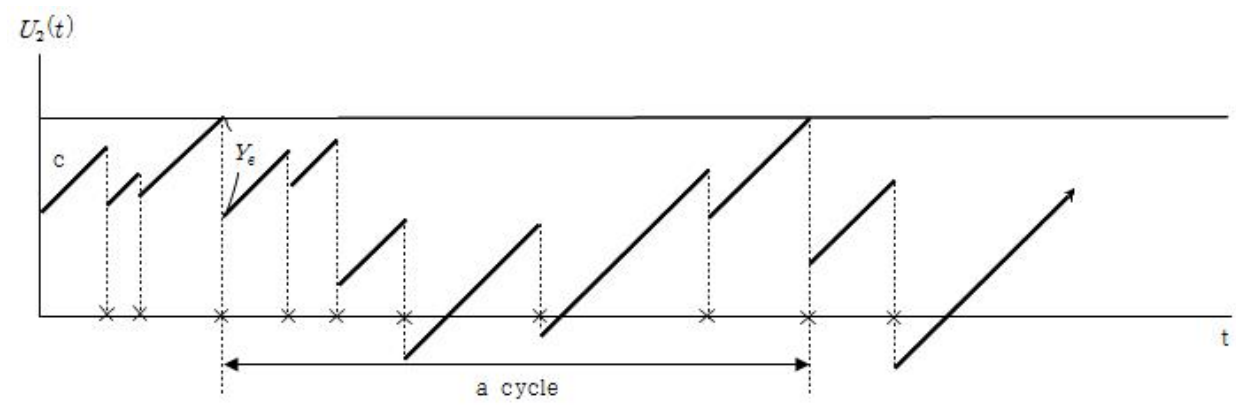

Figure 3: A sample path of $U_{2}(t)$.

the original process and connecting them together. $U_{2}(t)$ is similarly formed by separating the periods where $U(t) \leq V$ from the original process and connecting them together. $U_{1}(t)$ and $U_{2}(t)$ are illustrated in Figures 2 and 3, respectively. In the following subsections, we study the stationary distributions of $U_{1}(t)$ and $U_{2}(t)$ and, finally, the stationary distribution $F(x)$ of $U(t)$.

\subsection{Stationary distribution of $U_{1}(t)$}

Note that the time points where $U_{1}(t)=V$ form imbedded regeneration points of $\left\{U_{1}(t), t \geq 0\right\}$. Let $T$ denote the length of a cycle between two successive regeneration points, then it can be written as

$$
T=\inf \left\{t \geq 0: U_{1}(t) \notin[V, \infty)\right\}
$$

and $T$ is a stopping time with respect to $U_{1}(t)$. To obtain $E(T)$, we consider the following process in a cycle:

$$
M(t)=U_{1}(t)-E\left[U_{1}(t)\right]
$$


It can be shown that $M(t)$ is a martingale in a cycle with $E[M(t)]=0$. See Appendix. Applying the optional sampling theorem (Karlin and Taylor, 1975, pp. 257-262) to $M(t)$ with $T$, we can have the following proposition:

\section{Proposition 1.}

$$
E(T)=\frac{E\left(Y^{2}\right)}{2 \mu[\lambda \mu-(c-a)]} .
$$

Proof: We can show that in a cycle,

$$
E\left[U_{1}(t)\right]=V+(c-a) t-\lambda \mu t
$$

with $U_{1}(0)=V$. Since $T$ is the first time that $U_{1}(t)$ goes below $V$, the amount of drop below $V, Y_{e}$ say, will follow the equilibrium distribution of $G$, which is

$$
G_{e}(y)=\frac{1}{\mu} \int_{0}^{y}[1-G(x)] d x .
$$

Refer Klugman et al. (2004, p.240). Applying the optional sampling theorem to $M(t)$ with $T$ gives

$$
0=E[M(T)]=E\left[U_{1}(T)-\{V+(c-a) T-\lambda \mu T\}\right] .
$$

Since $E\left[U_{1}(T)\right]=V-E\left(Y_{e}\right)$ with $E\left(Y_{e}\right)=E\left(Y^{2}\right) / 2 \mu$, the result follows.

Let $F_{1}(x)$, for $x \geq V$, be the stationary distribution of $U_{1}(t)$. Since $U_{1}(t)$ is a regenerative process with cycles of length $T$, it is possible to obtain $F_{1}(t)$, if we know the probability that $U_{1}(t)$ reaches $x \geq V$ before it goes below $V$ and the expected duration that $U_{1}(t)$ stays over $x$ in a cycle. To do that, we consider the following process in a cycle:

$$
W(t)=\frac{e^{r U_{1}(t)}}{E\left[e^{r V(t)}\right]}, \quad \text { for }-\infty<r<\infty,
$$

where $V(t)=(c-a) t-\sum_{i=1}^{N(t)} Y_{i}$ and $\{N(t), t \geq 0\}$ is the arrival Poisson process of claims of rate $\lambda>0$. It can be shown that

$$
E\left[e^{r V(t)}\right]=\exp \left[r t(c-a)+\lambda t\left\{M_{Y}(-r)-1\right\}\right]
$$

where $M_{Y}(r)=\int_{0}^{\infty} e^{r y} d G(y)$ is the moment generating function of $Y$. Hence,

$$
W(t)=\exp \left[r U_{1}(t)-r t(c-a)-\lambda t\left\{M_{Y}(-r)-1\right\}\right] .
$$

It can be shown that $W(t)$ is a martingale in a cycle, for $-\infty<r<\infty$. See Appendix.

Proposition 2. Let $\theta$ be the solution of

$$
a(r)=r(c-a)+\lambda\left\{M_{Y}(-r)-1\right\}=0,
$$

then $\theta>0$ exists uniquely, when $0<c-a<\lambda \mu$. 
Proof: Note that $a(0)=0$ and $\lim _{r \rightarrow \infty} a(r)=\infty$. Moreover,

$$
a^{\prime}(r)=(c-a)-\lambda M_{Y}^{\prime}(-r) \quad \text { and } \quad a^{\prime \prime}(r)=\lambda M_{Y}^{\prime \prime}(-r)
$$

Since $a^{\prime}(0)=(c-a)-\lambda \mu<0$ and $a^{\prime \prime}(r)>0$, for all $r>0$, there exists a unique positive solution of $a(r)=0$.

Remark 1. When $Y$ is an exponential random variable with mean $\mu$,

$$
\theta=\frac{\lambda \mu-(c-a)}{(c-a) \mu}
$$

In summary, $W_{1}(t)=e^{\theta U_{1}(t)}$ is a martingale in a cycle, with $\theta>0$ satisfying $a(\theta)=0$.

For $x \geq V$, define the first exit time of $U_{1}(t)$, starting from $u(V \leq u \leq x)$, out of $[V, x)$ as

$$
T_{u}=\inf \left\{t \geq 0: U_{1}(t) \notin[V, x)\right\},
$$

then $T_{u}$ is a stopping time with respect to $U_{1}(t)$, and hence, with respect to $W_{1}(t)$. Applying the optional sampling theorem to $W_{1}(t)$ with $T_{u}$, we have the following proposition.

Proposition 3. Let $P_{x}^{u}$ be the probability that $U_{1}(t)$, starting from $u(V \leq u \leq x)$, reaches $x>V$ before it goes below $V$, and let $P_{V}^{u}$ be the probability that $U_{1}(t)$ goes below $V$ without reaching $x>V$, then

$$
P_{x}^{u}=\frac{e^{\theta u}-e^{\theta V} M_{Y e}(-\theta)}{e^{\theta x}-e^{\theta V} M_{Y e}(-\theta)}=1-P_{V}^{u}
$$

where $M_{Y_{e}}(-\theta)=\int_{0}^{\infty} e^{-\theta y} d G_{e}(y)=(1 / \mu \theta)\left[1-M_{Y}(-\theta)\right]$.

Proof: Applying the optional sampling theorem to $W_{1}(t)=e^{\theta U_{1}(t)}$ with $T_{u}$ gives

$$
\begin{aligned}
e^{\theta u} & =E\left[W_{1}\left(T_{u}\right)\right] \\
& =P\left[U_{1}\left(T_{u}\right)<V\right] E\left[W_{1}\left(T_{u}\right) \mid U_{1}\left(T_{u}\right)<V\right]+P\left[U_{1}\left(T_{u}\right)=x\right] E\left[W_{1}\left(T_{u}\right) \mid U_{1}\left(T_{u}\right)=x\right] \\
& =P_{V}^{u} E\left[e^{\theta\left(V-Y_{e}\right)}\right]+P_{x}^{u} e^{\theta x} \\
& =P_{V}^{u} \int_{0}^{\infty} e^{\theta(V-y)} d G_{e}(y)+P_{x}^{u} e^{\theta x} \\
& =P_{V}^{u} e^{\theta V} M_{Y_{e}}(-\theta)+P_{x}^{u} e^{\theta x} .
\end{aligned}
$$

Since $P_{x}^{u}+P_{V}^{u}=1$, the result follows, immediately.

\section{Remark 2.}

$$
P_{V}^{u}=1-P_{x}^{u}=\frac{e^{\theta x}-e^{\theta u}}{e^{\theta x}-e^{\theta V} M_{Y_{e}}(-\theta)} .
$$

Let $T(x)$ be the duration that $U_{1}(t)$, starting from $V$, stays over $x \geq V$ in a cycle. Observe that, once $U_{1}(t)$ crosses $x$, the duration that $U_{1}(t)$ stays over $x$, before it goes below $x$, is stochastically 
equal to $T$, for all $x \geq V$, since $U_{1}(t)$ satisfies the Markovian property in a cycle. Let $T_{1}, T_{2}, \ldots$ be a sequence of i.i.d. random variables having the same distribution as $T$, then,

$$
T(x)= \begin{cases}0, & \text { with probability } P_{V}^{V}, \\ T_{1}, & \text { with probability } P_{x}^{V} P_{V}^{x-Y_{e}}, \\ T_{1}+T_{2}, & \text { with probability } P_{x}^{V} P_{x}^{x-Y_{e}} P_{V}^{x-Y_{e}}, \\ \vdots & \\ T_{1}+T_{2}+\cdots+T_{n}, & \text { with probability } P_{x}^{V}\left(P_{x}^{x-Y_{e}}\right)^{n-1} P_{V}^{x-Y_{e}},\end{cases}
$$

where $P_{V}^{x-Y_{e}}=\bar{G}_{e}(x-V)+\int_{0}^{x-V} P_{V}^{x-y} d G_{e}(y)$, with $\bar{G}_{e}=1-G_{e}$, is the probability that $U_{1}(t)$, starting from $x$, goes below $V$ without crossing $x$ again, and $P_{x}^{x-Y_{e}}=\int_{0}^{x-V} P_{x}^{x-y} d G_{e}(y)$ is the probability that $U_{1}(t)$, starting from $x$, crosses $x$ again before it goes below $V$. Note that $P_{V}^{x-Y_{e}}+P_{x}^{x-Y_{e}}=1$.

Since $T(x)$ is a kind of geometric random variable, it can be show that

$$
E[T(x)]=E(T) \frac{P_{x}^{V}}{P_{V}^{x-Y_{e}}} .
$$

Finally, since $U_{1}(t)$ is a regenerative process with cycles of length $T$, by the renewal reward theorem of Ross (1996, pp. 133-135), the stationary distribution of $U_{1}(T)$ is given by

$$
\bar{F}_{1}(x)=\frac{E[T(x)]}{E(T)}=\frac{P_{x}^{V}}{P_{V}^{x-Y_{e}}}=1-F_{1}(x) .
$$

\subsection{Stationary distribution of $U_{2}(t)$}

The approach in Section 2.1 used to obtain the stationary distribution of $U_{1}(t)$ is not applicable in this section to obtain the stationary distribution of $U_{2}(t)$. Though $U_{2}(t)$ is also a regenerative process with cycles starting with $V-Y_{e}, U_{2}(t)$ passes $x \leq V$ down jump-wise, and hence, the amount of drop below $x$ is not stochastically specified and the expected duration that $U_{2}(t)$ stays below $x$ is hardly calculable. Therefore, we adopt a different approach, which is, establishing an integro-differential equation for the distribution function of $U_{2}(t)$ and solving the equation to obtain the moment generating function of the stationary distribution of $U_{2}(t)$.

Let $F_{2}(x, t)$ be the distribution function of $U_{2}(t)$, that is,

$$
F_{2}(x, t)=P\left\{U_{2}(t) \leq x\right\}, \quad \text { for }-\infty<x<V,
$$

and $F_{2}(x)$ be the stationary distribution function of $U_{2}(t)$. Conditioning on whether a claim arrives in a small interval $[t, t+\Delta t]$, we can have the following relations between $U_{2}(t)$ and $U_{2}(t+\Delta t)$ :

(i) If no claims arrive, then

$$
U_{2}(t+\Delta t)= \begin{cases}U_{2}(t)+c \Delta t, & \text { when } U_{2}(t) \leq V-c \Delta t \\ V-Y_{e}+c \Delta t, & \text { when } V-c \Delta t<U_{2}(t) \leq V .\end{cases}
$$

(ii) If a claim arrives, then

$$
U_{2}(t+\Delta t)=U_{2}(t)+c \Delta t-Y .
$$


Recall that the amount of drop below $V$ is equal to $Y_{e}$ and note that two or more claims arrive in a small interval $[t, t+\Delta t]$ is $o(\Delta t)$. Hence, we can obtain the following equation for $-\infty<x \leq V$,

$$
\begin{aligned}
P\left\{U_{2}(t+\Delta t) \leq x\right\}= & {[1-\lambda \Delta t+o(\Delta t)]\left[P\left\{U_{2}(t) \leq x-c \Delta t, U_{2}(t) \leq V-c \Delta t\right\}\right.} \\
& \left.+P\left\{V-Y_{e} \leq x-c \Delta t, V-c \Delta t<U_{2}(t) \leq V\right\}\right] \\
& +[\lambda \Delta t+o(\Delta t)] P\left\{U_{2}(t)-Y \leq x-c \Delta t\right\}+o(\Delta t) .
\end{aligned}
$$

Representing the equation for $F_{2}(x, t)$, we have

$$
\begin{aligned}
F_{2}(x, t+\Delta t)= & {[1-\lambda \Delta t+o(\Delta t)]\left[F_{2}(x-c \Delta t, t)+P\left\{V-Y_{e} \leq x-c \Delta t\right\}\left\{F_{2}(V, t)-F_{2}(V-c \Delta t, t)\right\}\right] } \\
& +[\lambda \Delta t+o(\Delta t)] P\left\{U_{2}(t)-Y \leq x-c \Delta t\right\}+o(\Delta t) .
\end{aligned}
$$

Here, applying Taylor series expansion on $F_{2}(x-c \Delta t, t)$ gives

$$
F_{2}(x-c \Delta t, t)=F_{2}(x, t)-c \Delta t \frac{\partial}{\partial x} F_{2}(x, t)+o(\Delta t)
$$

Observe also that conditioning on $Y$ yields

$$
\begin{aligned}
P\left\{U_{2}(t)-Y \leq x-c \Delta t\right\} & =\int_{0}^{\infty} F_{2}(x-c \Delta t+y, t) d G(y) \\
& =\int_{0}^{V-x+c \Delta t} F_{2}(x-c \Delta t+y, t) d G(y)+\bar{G}(V-x+c \Delta t)
\end{aligned}
$$

since $F_{2}(x, t)=1$ for $x \geq V$. Inserting these two equations into the above equation of $F_{2}(x-c \Delta t, t)$, we have

$$
\begin{aligned}
F_{2}(x, t+\Delta t)= & F_{2}(x, t)-c \Delta t \frac{\partial}{\partial x} F_{2}(x, t)-\lambda \Delta t F_{2}(x, t)+\bar{G}_{e}(V-x+c \Delta t)\left\{F_{2}(V, t)-F_{2}(V-c \Delta t, t)\right\} \\
& +\lambda \Delta t\left[\int_{0}^{V-x+c \Delta t} F_{2}(x-c \Delta t+y, t) d G(y)+\bar{G}(V-x+c \Delta t)\right]+o(\Delta t) .
\end{aligned}
$$

Subtracting $F_{2}(x, t)$ from both sides of the equation, dividing by $\Delta t$, and letting $\Delta t \rightarrow 0$, we have the following integro-differential equation for $F_{2}(x, t)$ :

$$
\frac{\partial}{\partial t} F_{2}(x, t)=-c \frac{\partial}{\partial x} F_{2}(x, t)-\lambda F_{2}(x, t)+c f_{2}(V, t) \bar{G}_{e}(V-x)+\lambda \int_{0}^{V-x} F_{2}(x+y, t) d G(y)+\lambda \bar{G}(V-x),
$$

where $f_{2}(V, t)=\left.(\partial / \partial x) F_{2}(x, t)\right|_{x=V}$.

Now, in the stationary case, $(\partial / \partial t) F_{2}(x, t)=0$, and hence, $F_{2}(x)$ satisfies

$$
0=-c \frac{\partial}{\partial x} F_{2}(x)-\lambda F_{2}(x)+c f_{2}(V) \bar{G}_{e}(V-x)+\lambda \int_{0}^{V-x} F_{2}(x+y) d G(y)+\lambda \bar{G}(V-x),
$$

where $f_{2}(V)=\left.(d / d x) F_{2}(x)\right|_{x=V}$. Let $M_{2}(r)=\int_{-\infty}^{V} e^{r x} d F_{2}(x)$ be the moment generating function of $F_{2}(x)$. Multiplying both sides of the above equation by $e^{r x}$ and taking Stieltjes integrals on both sides give

$$
0=-c\left[e^{r V} f_{2}(V)-r M_{2}(r)\right]-\lambda M_{2}(r)+c f_{2}(V) e^{r V} M_{Y_{e}}(-r)+\lambda M_{2}(r) M_{Y}(-r)
$$


where $M_{Y}(-r)=\int_{0}^{\infty} e^{-r y} d G(y)$ and $M_{Y_{e}}(-r)=\int_{0}^{\infty} e^{-r y} d G_{e}(y)=(1 / \mu r)\left[1-M_{Y}(-r)\right]$.

Solving the above equation for $M_{2}(r)$, we have

$$
M_{2}(r)=\frac{c e^{r V} f_{2}(V)\left[1-M_{Y_{e}}(-r)\right]}{c r-\lambda+\lambda M_{Y}(-r)} .
$$

Since $M_{2}(0)=1$, we can obtain $f_{2}(V)$ by applying l'Hôpital's rule, which is given by

$$
f_{2}(V)=\frac{c-\lambda \mu}{c E(Y)_{e}}=\frac{2 \mu(c-\lambda \mu)}{c E\left(Y^{2}\right)} .
$$

Finally, $M_{2}(r)$ is given by

$$
M_{2}(r)=\frac{2 e^{r V}(c-\lambda \mu)\left[\mu r-1+M_{Y}(-r)\right]}{r E\left(Y^{2}\right)\left[c r-\lambda+\lambda M_{Y}(-r)\right]} .
$$

\subsection{Stationary distribution of $U(t)$}

We, in this section, find the stationary distribution of the original process, $U(t)$, based on the results of Sections 2.1 and 2.2. Recall that $U_{1}(t)$ is a regenerative process with cycles of expected length

$$
E\left(T_{1}\right)=\frac{E\left(Y^{2}\right)}{2 \mu[\lambda \mu-(c-a)]} .
$$

Here, for the convenience of notation, we use $T_{1}$ instead of $T$.

Note that $U_{2}(t)$ is also a regenerative process with cycles starting when the surplus level drops below $V$ by an amount of $Y_{e}$ from $V$. Let $T_{2}$ denote the length of a cycle, then it can be written as

$$
T_{2}=\inf \left\{t \geq 0: U_{2}(t) \notin(-\infty, V]\right\}
$$

and $T_{2}$ is a stopping time with respect to $U_{2}(t)$. To obtain $E\left(T_{2}\right)$, we consider the following process in a cycle:

$$
L(t)=U_{2}(t)-E\left[U_{2}(t)\right]
$$

It can be shown that $L(t)$ is a martingale in a cycle with $E[L(t)]=0$. We omit the proof, since it is similar to that of showing $M(t)$ being a martingale. Applying the optional sampling theorem to $L(t)$ with $T_{2}$, we can have the following proposition:

\section{Proposition 4.}

$$
E\left(T_{2}\right)=\frac{E\left(Y^{2}\right)}{2 \mu(c-\lambda \mu)} .
$$

Proof: We can show that in a cycle,

$$
E\left[U_{2}(t)\right]=V-E\left(Y_{e}\right)+c t-\lambda \mu t
$$

with $U_{2}(0)=V-E\left(Y_{e}\right)$. Note that $T_{2}$ is the first time that $U_{2}(t)$ crosses $V$ in a cycle. Applying the optional sampling theorem to $L(t)$ with $T_{2}$ gives

$$
0=E\left[L\left(T_{2}\right)\right]=E\left[U_{2}\left(T_{2}\right)-\left\{V-E\left(Y_{e}\right)+c T_{2}-\lambda \mu T_{2}\right\}\right] .
$$


Since $E\left[U_{2}\left(T_{2}\right)\right]=V$ and $E\left(Y_{e}\right)=E\left(Y^{2}\right) / 2 \mu$, the result follows.

Finally, observe that the original process, $U(t)$, is also a regenerative process with cycles in which the cycles of $U_{1}(t)$ and $U_{2}(t)$ alternate with weights $E\left(T_{1}\right)$ and $E\left(T_{2}\right)$, which are the expected lengths of cycles of $U_{1}(t)$ and $U_{2}(t)$, respectively. Hence, from the renewal reward theorem of Ross (1996, pp. 133-135), the stationary distribution of $U(t)$ is given by

$$
F(x)=\frac{E\left(T_{1}\right) F_{1}(x)+E\left(T_{2}\right) F_{2}(x)}{E\left(T_{1}\right)+E\left(T_{2}\right)}, \text { for }-\infty<x<\infty,
$$

where $F_{2}(x)$ can be obtained from its moment generating function, $M_{2}(r)$, by an inversion.

Remark 3. It is not easy to invert $M_{2}(r)$ to find $F_{2}(x)$, theoretically, however, it will be possible to do, numerically, if the distribution function $G(y)$ and parameters, $c, \lambda$, and $V$, are specified, in practice.

\section{Appendix:}

Proof of $\boldsymbol{M ( t )}$ being a martingale: It is enough to show $E[M(t+s) \mid M(u), 0 \leq u \leq t]=M(t)$, for $s>0$, and $t>0$ :

$$
\begin{aligned}
& E[M(t+s) \mid M(u), 0 \leq u \leq t] \\
& =E\left[U_{1}(t+s)-E\left[U_{1}(t+s)\right] \mid U_{1}(u), 0 \leq u \leq t\right] \\
& =E\left[U_{1}(t+s) \mid U_{1}(u), 0 \leq u \leq t\right]-E\left[E\left[U_{1}(t+s)\right] \mid U_{1}(u), 0 \leq u \leq t\right] \\
& =E\left[U_{1}(t+s)-U_{1}(t) \mid U_{1}(u), 0 \leq u \leq t\right]+E\left[U_{1}(t) \mid U_{1}(u), 0 \leq u \leq t\right]-E\left[U_{1}(t+s)\right] \\
& =(c-a) s-\lambda \mu s+U_{1}(t)-\left\{(c-a) s-\lambda \mu s+E\left[U_{1}(t)\right]\right\} \\
& =U_{1}(t)-E\left[U_{1}(t)\right] \\
& =M(t) .
\end{aligned}
$$

Proof of $W(t)$ being a martingale: It is enough to show $E[W(t+s) \mid W(u), 0 \leq u \leq t]=W(t)$, for $s>0$, and $t>0$ :

$$
\begin{aligned}
& E[W(t+s) \mid W(u), 0 \leq u \leq t] \\
& =E\left[\exp \left[r U_{1}(t+s)-\lambda(t+s)\left\{M_{Y}(-r)-1\right\}-r(t+s)(c-a)\right] \mid W(u), 0 \leq u \leq t\right] \\
& =E\left[\exp \left[r U_{1}(t)-r \Sigma_{i=N(t)}^{N(t+s)} Y_{i}-\lambda t\left\{M_{Y}(-r)-1\right\}-\lambda s\left\{M_{Y}(-r)-1\right\}-r t(c-a)\right] \mid W(u), 0 \leq u \leq t\right] \\
& =E\left[\exp \left[r U_{1}(t)-\lambda t\left\{M_{Y}(-r)-1\right\}-r t(c-a)\right] \times \exp \left[-r \Sigma_{i=N(t)}^{N(t+s)} Y_{i}-\lambda s\left\{M_{Y}(-r)-1\right\} \mid W(u), 0 \leq u \leq t\right]\right] \\
& =W(t) e^{-\lambda s\left\{M_{Y}(-r)-1\right\}} E\left[\exp \left[-r \Sigma_{i=N(t)}^{N(t+s)} Y_{i} \mid W(u), 0 \leq u \leq t\right]\right], \quad \text { by independent increments } \\
& =W(t) e^{-\lambda s\left\{M_{Y}(-r)-1\right\}} E\left[\exp \left[-r \Sigma_{i=1}^{N(s)} Y_{i}\right]\right], \quad \text { by stationary increments } \\
& =W(t) e^{-\lambda s\left\{M_{Y}(-r)-1\right\}} e^{\lambda s\left\{M_{Y}(-r)-1\right\}} \\
& =W(t) .
\end{aligned}
$$




\section{References}

Cho EY, Choi SK, and Lee EY (2013). Transient and stationary analyses of the surplus in a risk model, Communications for Statistical Applications and Methods, 20, 475-480.

Dickson DCM and Willmot GE (2005). The density of the time to ruin in the classical Poisson risk model, ASTIN Bulletin, 35, 45-60.

Dufresne F and Gerber HU (1991). Risk theory for the compound Poisson process that is perturbed by diffusion, Insurance: Mathematics \& Economics, 10, 51-59.

Gerber HU (1990). When does the surplus reach a given target? Insurance: Mathematics \& Economics, 9, 115-119.

Gerber HU and Shiu ESW (1997). The joint distribution of the time of ruin, the surplus immediately before ruin, and the deficit at ruin, Insurance: Mathematics \& Economics, 21, 129-137.

Jeong MO and Lee EY (2010). Optimal control of the surplus in an insurance policy, Journal of the Korean Statistical Society, 39, 431-437.

Jeong MO, Lim KE, and Lee EY (2009). An optimization of a continuous time risk process, Applied Mathematical Modelling, 33, 4062-4068.

Karlin S and Taylor HM (1975). A First Course in Stochastic Processes (2nd ed.), Academic Press, New York.

Kim S and Lee EY (2015). Stationary distribution of the surplus in a risk model with dividends and reinvestments, Journal of the Korean Statistical Society, 44, 516-529.

Klugman SA, Panjer HH, and Willmot GE (2004). Loss Models: From Data to Decisions (2nd ed.), John Wiley \& Sons, Hoboken, NJ.

Ross SM (1996). Stochastic Processes (2nd ed.), John Wiley \& Sons, New York.

Song MJ, Kim JW, and Lee JY (2012). A compound Poisson risk model with variable premium rate, Journal of the Korean Data \& Information Science Society, 23, 1289-1297.

Won HJ, Choi SK, and Lee EY (2013). Ruin probabilities in a risk process perturbed by diffusion with two types of claims, Journal of the Korean Data \& Information Science Society, 24, 1-12. 